\title{
Carbon-Nanofibers-Based Micro-/Nanodevices for Neural-Electrical and Neural-Chemical Interfaces
}

\author{
Hongzhi Zhang, ${ }^{1}$ Mei Yu, ${ }^{1}$ Lei Xie, ${ }^{1}$ Linlin Jin, ${ }^{1}$ and Zhe $\mathrm{Yu}^{1,2,3}$ \\ ${ }^{1}$ Biomedical Microdevices Research Laboratory, Shenzhen Institutes of Advanced Technology, Chinese Academy of Sciences, \\ Shenzhen University Town, Shenzhen 518055, China \\ ${ }^{2}$ Key Laboratory for Health Informatics, Chinese Academy of Sciences, Shenzhen 518055, China \\ ${ }^{3}$ Shenzhen Key Laboratory of Neuropsychiatric Modulation, Shenzhen 518055, China \\ Correspondence should be addressed to Zhe Yu, zhe.yu@siat.ac.cn
}

Received 16 December 2011; Revised 24 February 2012; Accepted 24 February 2012

Academic Editor: Tong Lin

Copyright (C) 2012 Hongzhi Zhang et al. This is an open access article distributed under the Creative Commons Attribution License, which permits unrestricted use, distribution, and reproduction in any medium, provided the original work is properly cited.

\begin{abstract}
Carbon nanofibers (CNFs) have shown great potentials for development of micro-/nanodevices for neural interfaces due to their suitable properties, such as chemical stability, good electrical conductivity, ultramicro size with low electrical impedance, 3D structures with high surface-to-volume ratio, and long-term biocompatibility. In this paper, we review the applications of CNFs as neural-electrical interfaces and neural-chemical interfaces for neural recording and stimulation, electroconductive nanofibrous scaffolds for nerve tissue engineering, drug and gene delivery, and neurochemical sensing. The CNFs-based micro-/nanodevices provide new platforms to fine-tune electrical and chemical cues of neurons at subcellular nanoscale, which can be used for both fundamental studies of material-cell interactions and the development of chronically stable, implantable neural interface devices. Further development of this technology may potentially enable a highly multiplex closed-loop system with multifunctions for neuromodulation and neuroprostheses.
\end{abstract}

\section{Introduction}

In the past decade, nanotechnology has attracted so much attention and various nanomaterials and nanostructures have been developed, including quantum dots [1], nanofibers and nanotubes [2], nanowires [3, 4], and nanobelts [5]. These nanomaterials are of particular interest to neuroscience society because they can realize electrical and chemical communications with nervous system at micro- and nanoscale. Applications of nanostructures to neuroscience rapidly and widely expand from molecular imaging [6], scaffolds for neural regeneration [7], to neural interfaces [8, 9].

Neural interfaces create links and allow communications between the nervous system and the outside world by outputs of neural electrical and chemical signals for monitoring neural activities, inputs of neurostimulation and intracellular delivery of chemicals for modulating neural activities or outputs and inputs with feedbacks [10]. Neural interfaces have been used to record neural signals and treat or assist the patients with diseases or disabilities of neural function [11, 12]. Among these, electrical and chemical neural interfaces are widely used to input electrical and chemical stimulation to special part of the central or peripheral nervous system to help restore sensory processing or to improve function by modulating neural activities, and they are also used to record the electrical potentials or detect neural-chemicals to monitor ongoing neural activities. Micro-/nanodevices are designed and fabricated for electrical and chemical neural interfaces. In the conventional technologies for neural interfaces, metal and semiconductor bulk materials are commonly used.

Compared with the conventional technologies, carbon nanofibers (CNFs) have great potentials as electrical and chemical neural interfaces, for their superior electrical, chemical, and physical properties: (1) chemically stable and inert in physiological environment, (2) biocompatible for long-term implantation due to their covalent carbon structure, (3) electrically robust and conductive for 
signal detection, (4) 3D structures that allow intratissue and intracellular penetration, (5) with high surface-to-volume ratio, which reduces contacting electrical impedance greatly, and (6) high spatial resolution due to their ultramicro scale sizes. Here, we review the recent progress of applying CNFs to develop informative neural interfaces, including neuralelectrical interfaces for recording and stimulation of neural activities, electroconductive nanofibrous scaffolds, and neural-chemical interfaces for minimally invasive molecular delivery and detection of neurochemicals.

\section{Neural-Electrical Interfaces}

Neural-electrical interfaces are widely used for bidirectional communications between the nervous system and external devices, which detect and modulate neural functions by neural recording and stimulation for diagnosis and treatment of neurological diseases or artificial limb control. High spatial resolution of information communication is important for neural decoding and modulation of specific targets. However, submicron-scale planar electrodes are hardly able to detect neural signals because double-layer impedance of the electrodes in physiological solutions rapidly increases as the size of individual electrode is scaled down. Given the $3 \mathrm{D}$ structures with high surface-to-volume ratio, $\mathrm{CNFs}$ electrodes can provide a large active surface area for neural recording and stimulation while individual electrode sites on the substrate are scaled down.

For both functional electrical stimulation (FES) and neural recording, a stable, reliable, and biocompatible neuralelectrical interface is essential, which requires safe and effective delivery of electrical charges between the nervous system and electrodes for a relatively long term. Currently, most traditional microelectrodes are fabricated with rigid metals and semiconductors [13, 14]. Compared to metal-based microelectrode arrays (MEAs), CNFs have two superior characteristics to be employed in the neural-electrical interfaces. Firstly, CNFs can provide a high resolution. There are some difficulties to improve the resolution of conventional metal-based MEAs, because reducing the size of metal-based microelectrode increases the electrode impedance and thermal noise, which compromises the sensibility of electrodes to detect electrical signals from the nervous system $[15,16]$. However, CNFs can provide a large active surface area with small sites on the substrate allowing for a higher spatial resolution. Secondly, CNFs not only can work at the extracellular level but also may penetrate into neurons and then work at the intracellular level. On the surface of neural tissues in vivo or tissue slices in vitro, there is a glia layer accompanied by some injured and dead cells. The planar-metal-based electrodes cannot penetrate the unexpected layer to active cells. The neural signal attenuates greatly through the glia layer and some of the signals from deep inside the tissue are hard to detect. CNFs, especially vertical aligned carbon nanofibers (VACNFs), are of conical structures with tiny tips, and they have the ability to penetrate through the glia layer into tissues. Taking VACNFs for example, Figure 1(a1) shows a simple schematic of a bidirectional neural-electrical interface platform. "Bidirectional" means this platform can both input electrical stimulation and record neural electrical potentials at the same time, which realizes a real-time monitoring of neural electrical activities and electrical modulating stimulation simultaneously.

Researchers in NASA Ames Research Center (NARC, USA) reported their a series of advancements in developing 3D brush-like VACNFs [17-19]. They fabricated VACNFs on a silicon wafer by plasma-enhanced chemical vapor deposition using $\mathrm{Ni}$ as catalyst and tested them on PC12 cells. The results indicated that the soft 3D VACNFs architecture provided a new platform to fine-tune the topographical, mechanical, chemical, and electrical cues at subcellular nanoscale. Furthermore, they developed polypyrrole ( $\mathrm{PPy}-)$ coated VACNFs, which showed enhanced capability of neural stimulation [17]. Researchers in Columbia University (USA) and Oak Ridge National Laboratory (ORNL, USA) developed a CNFs-based neural chip and demonstrated its capability of both stimulating and recording electrophysiological signals from brain tissues in vitro $[20,21]$. In this study, long-term potentiation (LTP) was induced and detected through CNFs arrays. Researchers in the University of Tennessee (USA) developed thin-film transistor (TFT-) VACNFs [22]. They fabricated the VACNFs on an active matrix TFT and demonstrated a novel MEA platform. By using this new platform, stimulating and recording could also be realized simultaneously. VACNFs integrated on the TFT array enhanced the electrical selectivity to the cell, and furthermore, they provided the potential for intracellular sensing within individual cells.

In addition, carbon nanofibrous materials have been developed as electroconductive nanofibrous scaffolds for neural tissues to facilitate communication through neural interfaces. Electrical fields are able to enhance and direct nerve growth [23], therefore electroconductive scaffolds are applied to enhance the nerve regeneration process, not only providing physical support for cell growth but also delivering the functional stimulus (Figure $1(\mathrm{a} 2)$ ). In recent studies, electrospun biocompatible, and electroconductive polymer nanofibers were widely investigated, such as polyaniline $(\mathrm{PANI}) /$ poly $(\varepsilon$-caprolactone)/gelatin (PG) [24], polypyrrole (PPy)/poly(styrene- $\beta$-isobutylene- $\beta$-styrene) (SIBS) $[25,26]$, PPy/poly(lactic coglycolic acid) (PLGA) [27], and their variants. The electroconductive polymer fibrous scaffolds has shown to be qualified for cell attachment and proliferation [25-27]. Moreover, electrical stimulation through conductive nanofibrous scaffolds have shown their capability of enhancing cell proliferation and neuite outgrowth compared to the scaffolds that were not subjected to electrical stimulation [24].

Carbon nanomaterials are naturally good electrical conductor and have good biocompatibility, which make them good candidates for electrically conductive scaffolds [28]. Researchers in Regenerative Medicine Institute in National University of Ireland reported their study of carbon nanotube(CNT)-based poly (L-lactic acid) (PLA) nanofibers scaffolds. Compared to PLA nanofiber scaffolds, the presence of CNT- enhanced electrical conductivity and physical stability of the scaffolds with less degradation visible on the surface 
(a) Neural-electrical interfaces

(a1)

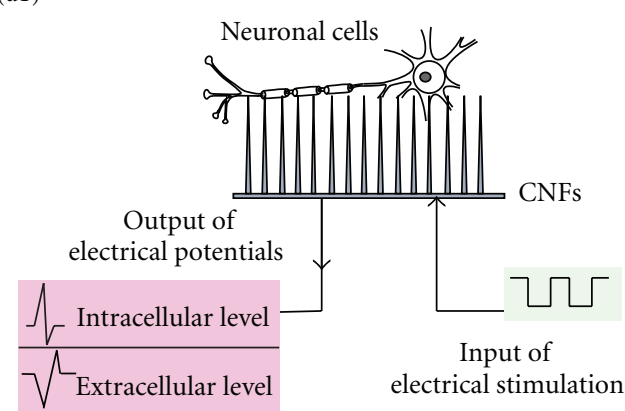

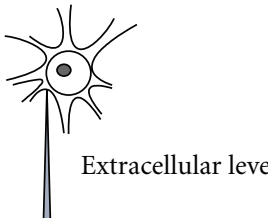

(a2)

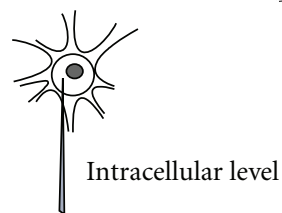

(b) Neural-chemical interfaces
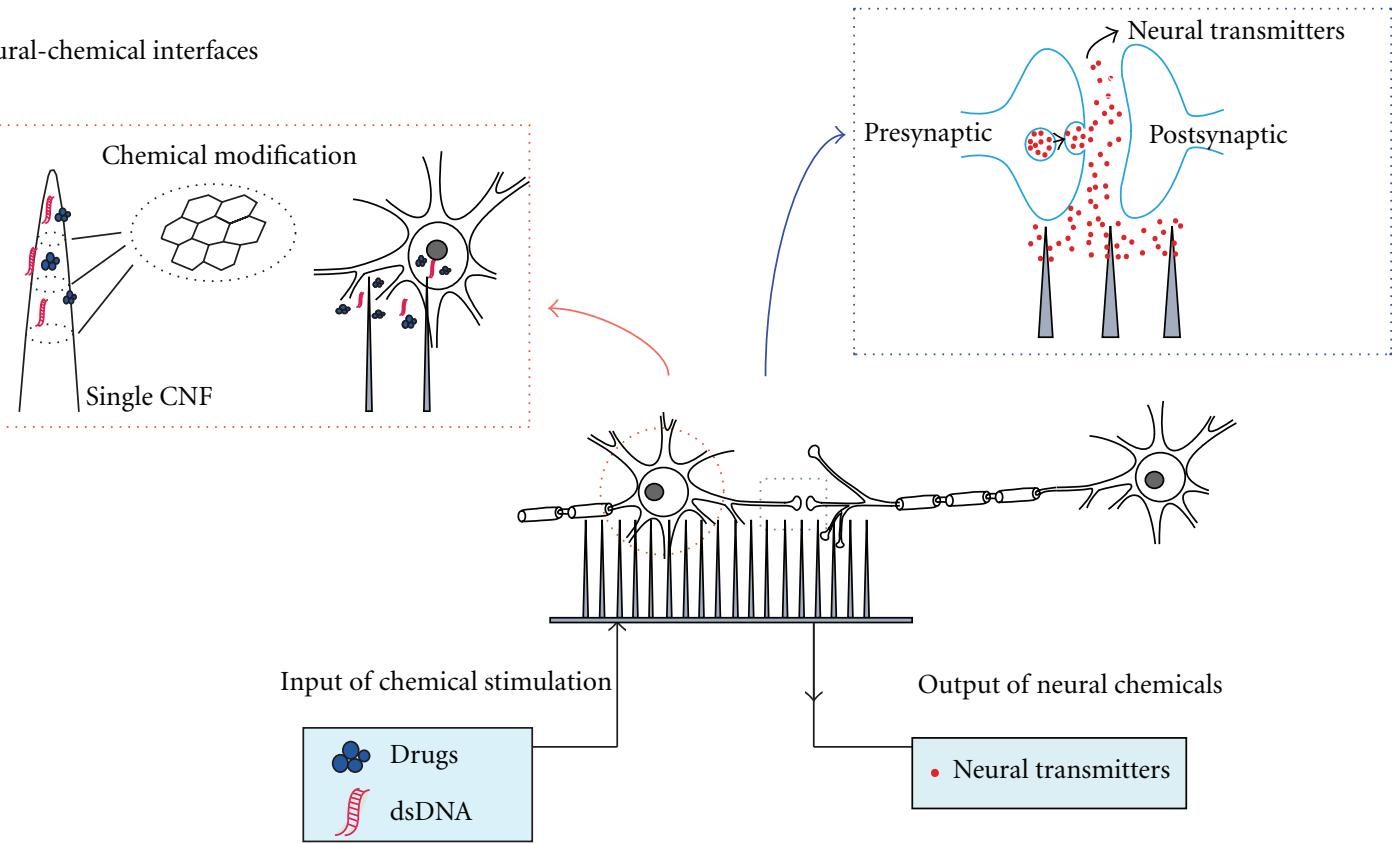

III $\mid$ || $\mid$ ||

electrical modulation 
Drugs and therapeutic genes are sent or locally released to a desirable group of target cells to treat neurological diseases [31, 32]. Although microinjection is widely used for drug delivery [33], the microneedle may cause damage to cell membranes. Recently, VACNFs were found capable of penetrating through cell membranes to deliver drugs and genes $[34,35]$. Drugs and genes were first attached to VACNFs by either covalent or noncovalent bonding, and then the drug/gene carrier conjugates were directly injected into the target cells. By using this method, the cell membrane disruption was minimized due to cell endocytosis of the ultramicro CNF tip. And also, this method could deliver drugs or DNAs to a large number of cells simultaneously by a bundle-located CNFs. Researchers in ORNL (USA) reported a strategy for delivering double-stranded DNA (dsDNA) into cells. The dsDNA was covalently and end specifically bounded onto VACNFs and then released after VACNFs penetrating the cell membranes. The whole process was tested on Chinese hamster lung epithelial cells, and the result indicated that this approach enabled the transport of bound ligands directly into cell nuclei and bypassed extracellular and cytosolic degradation. Till now, few researches on using CNFs for delivering drugs or genes to treat neurological diseases have been reported, whereas the successful delivery of dsDNA by CNFs indicates potential applications of CNFs as neural-chemical interfaces to the gene therapies for neurological diseases.

As for the detection of neural-chemicals, single carbon fiber electrode has been widely used [36]. However, this kind of electrode is designed for recording of neurochemicals from single site rather than neural networks. One group in NARC (USA) reported improvement of using VACNFs as multiplexing biosensors $[37,38]$. The devices were developed to detect the DNA targets that corresponded to E. coli O157:H7 $16 \mathrm{~S}$ rRNA and be able to produce reliable electrochemical responses with high signal-to-noise ratio. This technology showed important progresses for chemical sensing with well-controlled radial diffusion transport, minimal background currents and increased sensitivity. Another group in NARC (USA) and in Department of Neurologic Surgery (USA) reported integration of the CNFs electrode arrays with wireless instantaneous neurotransmitter concentration sensor system to detect dopamine by using fast scan cyclic voltammetry [39]. The CNFs facilitated the detection of neurochemical release over a large area, yet with high spatial resolution. This technology enabled CNFs-based electrode arrays to simultaneously monitor electrical and chemical activities from the nervous system.

\section{Challenges and Outlook}

Both neural-electrical and neural-chemical interfaces provide tools for recording neural activities from the nervous system and delivering therapeutic elements to restore and recover neural function from diseases or injuries. In this paper, the recent applications of CNFs for interfacing with neurological system are reviewed. CNFs can provide high spatial resolution, high sensitivity, and minimal damage to neural tissue. As for clinical application as neural interfaces, the following issues needs further studying. (1) The biocompatibility of the materials that are introduced during CNFs fabrication (such as catalysts) need to be carefully investigated, and adjustment of fabrication process may be implemented if necessary. (2) The nucleation and growth mechanisms of CNFs are still not very clear, therefore, the homogeneity and yield of CNFs are unsatisfactory. (3) Some potential side effects accompanying CNFs-based neural interfaces exist. Because of their surface properties and atom structures, toxic and unexpected chemical compounds may bond and be delivered to the cell together with the useful ones. Therefore, the selectivity of CNFs needs further studying. (4) As electroconductive scaffolds, the interaction mechanism between CNFs and neuronal cells is not very clear, and further research is needed in order to control the nerve regeneration process better.

The CNFs have great potentials as multiplexing neural interfaces and intracellular neural interfaces, which are capable of dual-mode detecting electrophysiological and neurochemical signals, not only at the extracellular level with high spatial resolution, but also at the intracellular level by penetrating into single neurons. In the future, it is a trend to integrate neural-electrical interfaces and neural-chemical interfaces together as an intelligent, closed-loop therapeutic device for diagnosis and treatment of neurological diseases, realizing automatic modulation of neural activity by neurostimulation or local drug delivery responding to real-time detection of electrical and chemical information from the nervous system.

\section{Acknowledgments}

This work was supported by Grant 61102042 to Z. Y. from National Natural Science Foundation of China and Youth Innovation Promotion Association of Chinese Academy of Sciences.

\section{References}

[1] G. A. Posthuma-Trumpie, J. H. Wichers, M. Koets, L. B.J.M. Berendsen, and A. Van Amerongen, "Amorphous carbon nanoparticles: a versatile label for rapid diagnostic (immuno)assays," Analytical and Bioanalytical Chemistry, vol. 402, no. 2, pp. 593-600, 2012.

[2] L. Zhang, L. Chen, T. Wells, and M. El-Gomati, "Bamboo and herringbone shaped carbon nanotubes and carbon nanofibres synthesized in direct current-plasma enhanced chemical vapour deposition," Journal of Nanoscience and Nanotechnology, vol. 9, no. 7, pp. 4502-4506, 2009.

[3] M. F.L. De Volder, R. Vansweevelt, P. Wagner, D. Reynaerts, C. Van Hoof, and A. J. Hart, "Hierarchical carbon nanowire microarchitectures made by plasma-assisted pyrolysis of photoresist," ACS Nano, vol. 5, no. 8, pp. 6593-6600, 2011.

[4] X. Cao, Q. He, W. Shi et al., "Graphene oxide as a carbon source for controlled growth of carbon nanowires," Small, vol. 7, no. 9, pp. 1199-1202, 2011.

[5] W. Kubo, H. Hayakawa, K. Miyoshi, and S. Fujikawa, "Sizecontrolled simple fabrication of free-standing, ultralong metal 
nanobelt array," Journal of Nanoscience and Nanotechnology, vol. 11, no. 1, pp. 131-137, 2011.

[6] K. Seekell, M. J. Crow, S. Marinakos, J. Ostrander, A. Chilkoti, and A. Wax, "Hyperspectral molecular imaging of multiple receptors using immunolabeled plasmonic nanoparticles," Journal of Biomedical Optics, vol. 16, Article ID 116003, 2011.

[7] J. Y. Lee, C. A. Bashur, A. S. Goldstein, and C. E. Schmidt, "Polypyrrole-coated electrospun PLGA nanofibers for neural tissue applications," Biomaterials, vol. 30, no. 26, pp. 4325-4335, 2009.

[8] O. Onaca, R. Enea, D. W. Hughes, and W. Meier, "Stimuliresponsive polymersomes as nanocarriers for drug and gene delivery," Macromolecular Bioscience, vol. 9, no. 2, pp. 129-139, 2009.

[9] J. H. Kim, G. Kang, Y. Nam, and Y. K. Choi, "Surfacemodified microelectrode array with flake nanostructure for neural recording and stimulation," Nanotechnology, vol. 21, no. 8, Article ID 085303, 2010.

[10] N. G. Hatsopoulos and J. P. Donoghue, "The science of neural interface systems," Annual Review of Neuroscience, vol. 32, pp. 249-266, 2009.

[11] W. Wang, J. L. Collinger, M. A. Perez et al., "Neural interface technology for rehabilitation: exploiting and promoting neuroplasticity," Physical Medicine and Rehabilitation Clinics of North America, vol. 21, no. 1, pp. 157-178, 2010.

[12] S.-P. Kim, J. D. Simeral, L. R. Hochberg, J. P. Donoghue, G. M. Friehs, and M. J. Black, "Point-and-click cursor control with an intracortical neural interface system by humans with tetraplegia," IEEE Transactions on Neural Systems and Rehabilitation Engineering, vol. 19, no. 2, pp. 193-203, 2011.

[13] E. Azemi, G. T. Gobbel, and X. T. Cui, "Seeding neural progenitor cells on silicon-based neural probes: laboratory investigation," Journal of Neurosurgery, vol. 113, no. 3, pp. 673$681,2010$.

[14] J. Persson, N. Danielsen, and L. Wallman, "Porous silicon as a neural electrode material," Journal of Biomaterials Science, Polymer Edition, vol. 18, no. 10, pp. 1301-1308, 2007.

[15] J. J. Pancrazio, "Neural interfaces at the nanoscale," Nanomedicine, vol. 3, no. 6, pp. 823-830, 2008.

[16] W. Franks, I. Schenker, P. Schmutz, and A. Hierlemann, "Impedance characterization and modeling of electrodes for biomedical applications," IEEE Transactions on Biomedical Engineering, vol. 52, no. 7, pp. 1295-1302, 2005.

[17] E. D. de Asis, T. D. B. Nguyen-Vu, P. U. Arumugam et al., "High efficient electrical stimulation of hippocampal slices with vertically aligned carbon nanofiber microbrush array," Biomedical Microdevices, vol. 11, no. 4, pp. 801-808, 2009.

[18] T. D. B. Nguyen-Vu, H. Chen, A. M. Cassell, R. J. Andrews, M. Meyyappan, and J. Li, "Vertically aligned carbon nanofiber architecture as a multifunctional 3-D neural electrical interface," IEEE Transactions on Biomedical Engineering, vol. 54, no. 6, pp. 1121-1128, 2007.

[19] T. D. Nguyen-Vu, H. Chen, A. M. Cassell, R. Andrews, M. Meyyappan, and J. Li, "Vertically aligned carbon nanofiber arrays: an advance toward electrical-neural interfaces," Small, vol. 2, no. 1, pp. 89-94, 2006.

[20] Z. Yu, T. E. McKnight, M. N. Ericson, A. V. Melechko, M. L. Simpson, and B. Morrison, "Vertically aligned carbon nanofiber arrays record electrophysiological signals from hippocampal slices," Nano Letters, vol. 7, no. 8, pp. 2188-2195, 2007.

[21] Z. Yu, "Vertically aligned carbon nanofibre neural chip for interfacing with neurological system," in Proceedings of the IEEE International Conference on Nano/Molecular Medicine and Engineering, pp. 188-191, Hong Kong/Macau, China, December 2010.

[22] J. Park, S. Kwon, S. I. Jun et al., "Active-matrix microelectrode arrays integrated with vertically aligned carbon nanofibers," IEEE Electron Device Letters, vol. 30, no. 3, pp. 254-257, 2009.

[23] C. D. McCaig, A. M. Rajnicek, B. Song, and M. Zhao, "Controlling cell behavior electrically: current views and future potential," Physiological Reviews, vol. 85, no. 3, pp. 943-978, 2005.

[24] L. Ghasemi-Mobarakeh, M. P. Prabhakaran, M. Morshed, M. H. Nasr-Esfahani, and S. Ramakrishna, "Electrical stimulation of nerve cells using conductive nanofibrous scaffolds for nerve tissue engineering," Tissue Engineering A, vol. 15, no. 11, pp. 3605-3619, 2009.

[25] Y. Liu, X. Liu, J. Chen, K. J. Gilmore, and G. G. Wallace, "3D Bio-nanofibrous PPy/SIBS mats as platforms for cell culturing," Chemical Communications, no. 32, pp. 3729-3731, 2008.

[26] X. Liu, J. Chen, K. J. Gilmore, M. J. Higgins, Y. Liu, and G. G. Wallace, "Guidance of neurite outgrowth on aligned electrospun polypyrrole/ poly(styrene- $\beta$-isobutylene- $\beta$-styrene) fiber platforms," Journal of Biomedical Materials Research A, vol. 94, no. 4, pp. 1004-1011, 2010.

[27] J. Y. Lee, C. A. Bashur, A. S. Goldstein, and C. E. Schmidt, "Polypyrrole-coated electrospun PLGA nanofibers for neural tissue applications," Biomaterials, vol. 30, no. 26, pp. 43254335, 2009.

[28] P. R. Supronowicz, P. M. Ajayan, K. R. Ullmann, B. P. Arulanandam, D. W. Metzger, and R. Bizios, "Novel currentconducting composite substrates for exposing osteoblasts to alternating current stimulation," Journal of Biomedical Materials Research, vol. 59, no. 3, pp. 499-506, 2002.

[29] J. N. Mackle, D. J.-P. Blond, E. Mooney et al., "In vitro characterization of an electroactive carbon-nanotube-based nanofiber scaffold for tissue engineering," Macromolecular Bioscience, vol. 11, no. 9, pp. 1272-1282, 2011.

[30] G.-Z. Jin, M. Kim, U. S. Shin, and H.-W. Kim, "Neurite outgrowth of dorsal root ganglia neurons is enhanced on aligned nanofibrous biopolymer scaffold with carbon nanotube coating," Neuroscience Letters, vol. 501, no. 1, pp. 10-14, 2011.

[31] S. Haque, S. Md, M. I. Alam, J. K. Sahni, J. Ali, and S. Baboota, "Nanostructure-based drug delivery systems for brain targeting," Drug Development and Industrial Pharmacy, vol. 38, no. 4, pp. 387-411, 2012.

[32] X. Liu, "Clinical trials of intranasal delivery for treating neurological disorders a critical review," Expert Opinion on Drug Delivery, vol. 8, no. 12, pp. 1681-1690, 2011.

[33] D. V. McAllister, M. G. Allen, and M. R. Prausnitz, "Microfabricated microneedles for gene and drug delivery," Annual Review of Biomedical Engineering, vol. 2, no. 2000, pp. 289313, 2000.

[34] D. B. Peckys, A. V. Melechko, M. L. Simpson, and T. E. McKnight, "Immobilization and release strategies for DNA delivery using carbon nanofiber arrays and self-assembled monolayers," Nanotechnology, vol. 20, no. 14, Article ID 145304, 2009.

[35] D. B. Peckys, N. De Jonge, M. L. Simpson, and T. E. McKnight, "End-specific strategies of attachment of long double stranded DNA onto gold-coated nanofiber arrays," Nanotechnology, vol. 19, no. 43, Article ID 435301, 2008.

[36] X. Zhao, X. Lu, W. T. Y. Tze, and P. Wang, "A single carbon fiber microelectrode with branching carbon nanotubes for bioelectrochemical processes," Biosensors and Bioelectronics, vol. 25, no. 10, pp. 2343-2350, 2010. 
[37] P. U. Arumugam, H. Chen, S. Siddiqui et al., "Wafer-scale fabrication of patterned carbon nanofiber nanoelectrode arrays: a route for development of multiplexed, ultrasensitive disposable biosensors," Biosensors and Bioelectronics, vol. 24, no. 9, pp. 2818-2824, 2009.

[38] P. U. Arumugam, E. Yu, R. Riviere, and M. Meyyappan, "Vertically aligned carbon nanofiber electrode arrays for nucleic acid detection," Chemical Physics Letters, vol. 499, no. 4-6, pp. 241-246, 2010.

[39] J. E. Koehne, M. Marsh, A. Boakye et al., "Carbon nanofiber electrode array for electrochemical detection of dopamine using fast scan cyclic voltammetry," Analyst, vol. 136, no. 9, pp. 1802-1805, 2011. 

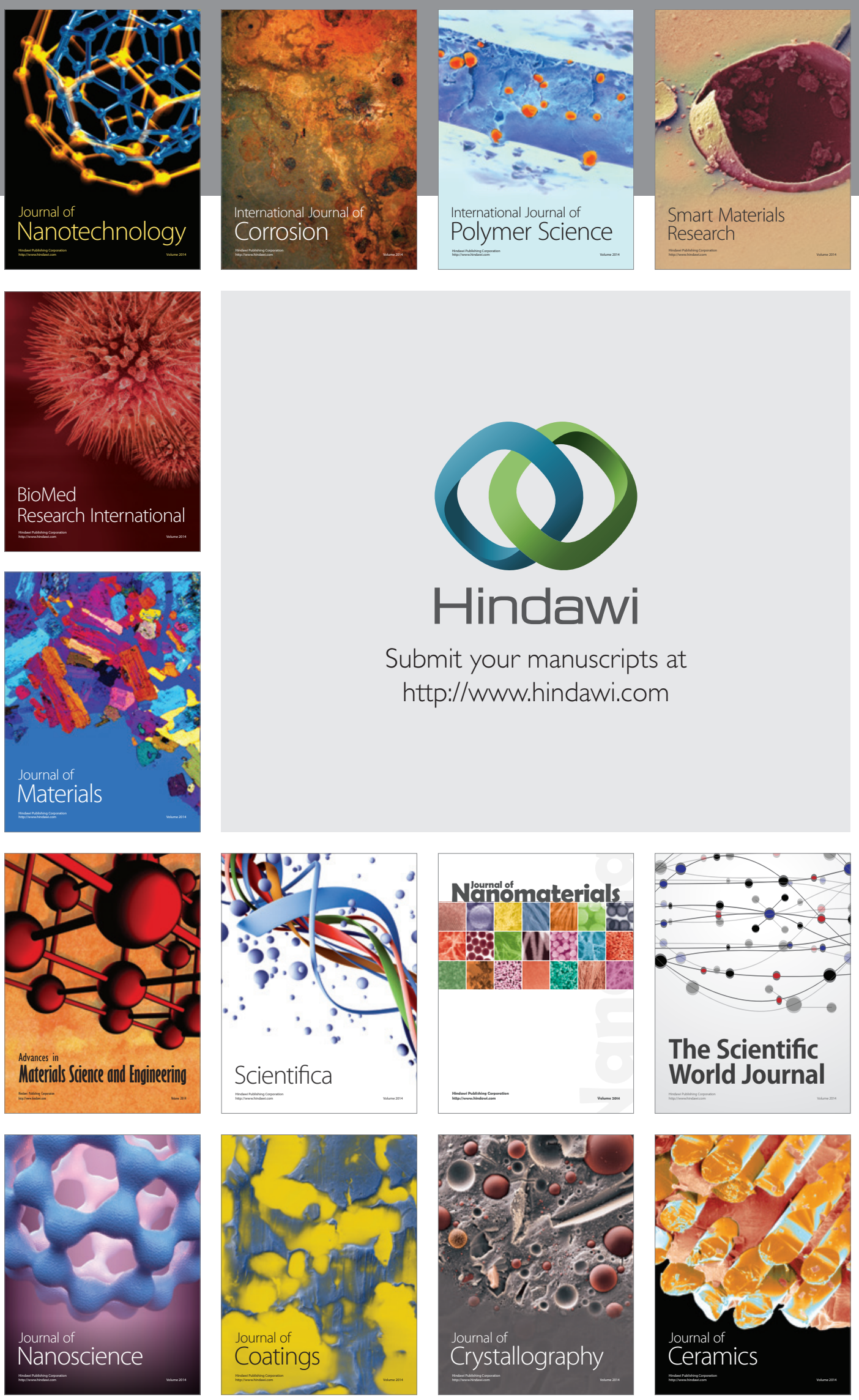

The Scientific World Journal

Submit your manuscripts at

http://www.hindawi.com

\section{World Journal}

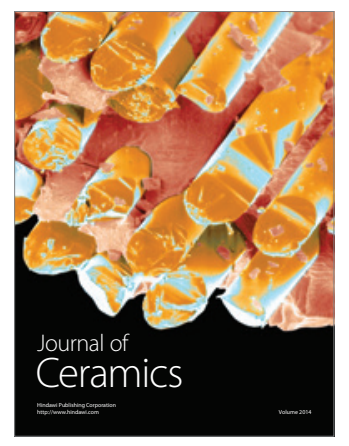

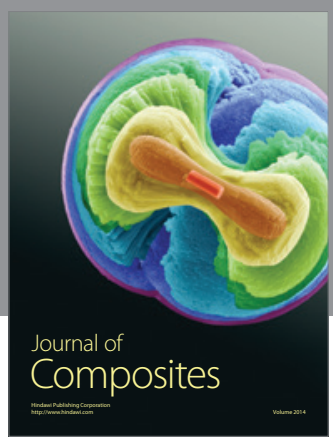
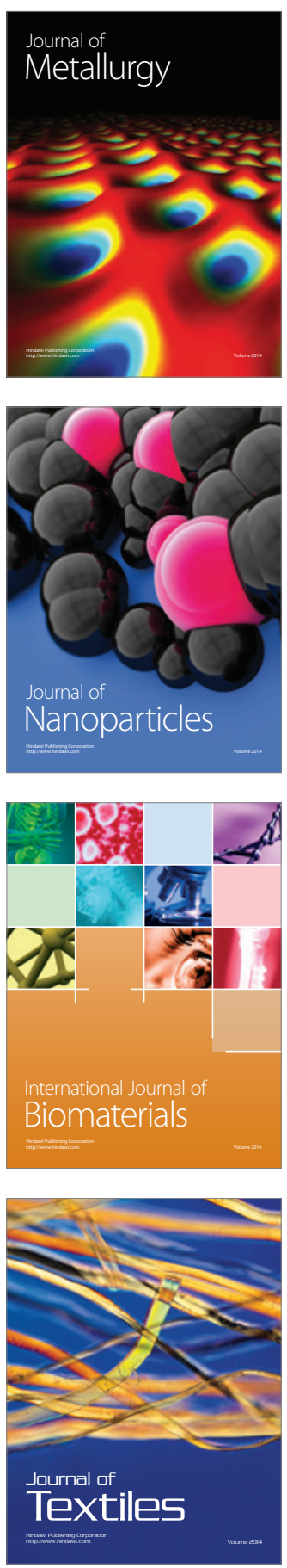